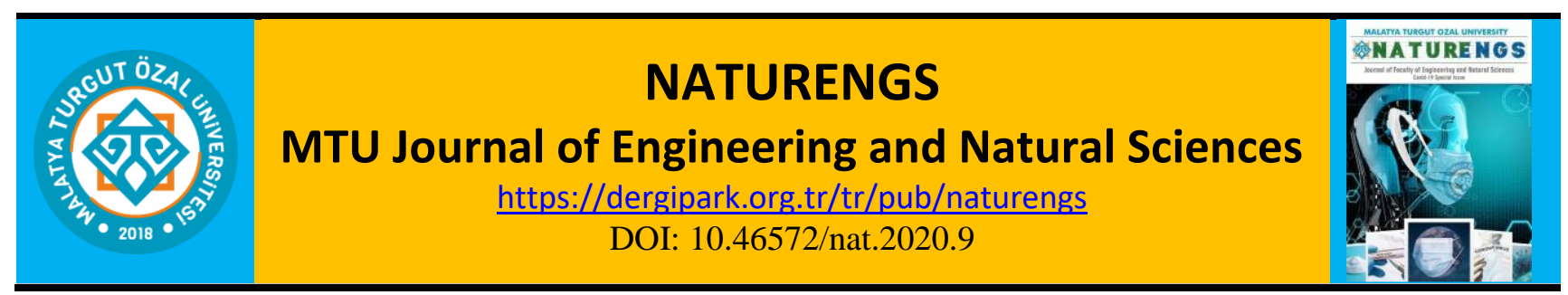

\title{
A Study on Foods That Boost The Immune System During The Covid-19 Pandemic
}

\author{
Ayse Biçer \\ Department of BioEngineering, Faculty of Engineering and Natural Sciences, Malatya Turgut Özal University, \\ Malatya, Turkey.
}

(Received: 05.10.2020; Accepted: 15.10.2020)

\begin{abstract}
The coronavirus (COVID-19) has spread rapidly across the world and has been declared a pandemic by the World Health Organization. One of the ways to combat COVID-19 and other infectious diseases is to boost the immune system. It is, therefore, of utmost importance to eat well and consume foods that boost the immune system. This study investigated the effect of Nigella sativa (black seed) oil, silverberry, date, grape, Asphodelus aestivus, Rheum ribes, and carob on the immune system. There is no cure yet for Covid-19. However, scientists and healthcare professionals recommend that people eat foods that are rich in vitamins and minerals to bolster their immune system to fight COVID-19 because immunodeficiency is the Achilles' heel of the Covid-19 pandemic
\end{abstract}

Keywords: Immune system, foods, metabolism, Covid-19, Thymoquinone.

\section{INTRODUCTION}

The coronavirus disease 2019 (COVID-19) was first reported at a local seafood wholesale market in Huanan, China, and since then has caused millions of infections and thousands of deaths worldwide. COVID-19, which causes severe acute respiratory distress syndrome, was declared a pandemic by the World Health Organization (WHO) on March 11, 2020 [1,2].

Clinical trials are currently underway worldwide to develop a vaccine against COVID-19, and infected individuals and those at the highest risk for infection are put in quarantine to help prevent the spread of the virus. The Centers for Disease Control and Prevention (CDC) recommend that all healthcare professionals wear standard protective equipment $[3,4]$.

COVID-19 spreads rapidly through respiratory droplets from person to person, including those with no link to the disease epicenter. The continuing increase in the number of COVID cases shows that community transmission is taking place worldwide [5].

There are several risk factors for disease progression in COVID-19 patients. Liver, kidney, and cardiovascular diseases, as well as tobacco use, are associated with increased severity and mortality from COVID-19 [6].

People with a weakened immune system are at higher risk for contracting Covid-19, like all viral infections. Immunodeficiency makes the body vulnerable to diabetes and cancer, as well as viral infections [7]. Protein-energy malnutrition (PEM) decreases immunity and increases

*Corresponding Author: ayse.bicer@ozal.edu.tr

ORCID number of authors: 0000 -0003-4514-5644 
the risk of contracting infectious diseases. Ritz and Gardner (2006) reported that PEM exacerbated by aging resulted in an increase in mortality from influenza infection, which they believed was due to low body weight reducing the availability of energy and immune function [8].

Current Covid-19 treatments focus mainly on preventing symptoms and complications. Therefore, the most crucial strategy, for now, is prevention. These strategies are designed to reduce exposure and boost immunity. Research shows that a healthy diet boosts the immune system [9].

It is necessary to know the immune system well before focusing on foods that boost it. The immune system consists of organs, cells, tissues, and proteins, which are responsible for bodily processes that combat viruses, bacteria, and pathogens that cause infections and diseases. The immune system is the collection of all processes by which pathogens and tumor cells are recognized and destroyed to protect the living organism against diseases. The immune system scans every foreign substance that enters or comes into contact with the body and distinguishes them from healthy body cells and tissues.

All foreign molecules that stimulate the immune system are referred to as "antigens" or "immunogens." Antigens are kept away from the body by a layered protective system consisting of surface barriers and the innate and adaptive immune system. Antigens can penetrate the first line of defense (skin, respiratory, and digestive system) and stimulate the second line of defense. The innate immune system consists of specialized centers, such as bone marrow, the thymus, the spleen, and lymph nodes. Defense cells and molecules (phagocytes, macrophages, and lymphocytes) involved in innate immunity come into play to combat the antigens that evade the surface barriers. First, phagocytes and macrophages, which are precursor cells, try to destroy the antigens. The body continually recognizes and destroys the foreign invaders that penetrate the first line of defense [10].

$\mathrm{B}$ and $\mathrm{T}$ lymphocytes are the primary cells of the adaptive immune system. They are activated in cases where the second line of defense fails, which triggers a rather complex chain system. $\mathrm{T}$ cells that detect antigens trigger other defense cells and numerous related biochemical cascades [11]. Malignant T cells, a subset of T cells, destroy antigens, while B cells begin to synthesize antibodies (immunoglobulins), known as smart molecules [12].

\section{BOOSTING THE IMMUNE SYSTEM}

Immunodeficiency is caused by the failure of one or more than one component of the immune system. Immunosenescence leads to a diminished response of the immune system to pathogens in both young and old populations, but especially after the age of 50. [13,14]. In developed countries, immunodeficiency is mainly caused by malnutrition as well as obesity, alcoholism, and illegal substance use. Protein-energy malnutrition (PEM) is associated with a serious deterioration in cell-mediated immunity, and the complement system, phagocytic functionality, change in IgA antibody concentrations, and cytokine production. Single nutrient deficiency (iron, copper, zinc, selenium, and vitamins A, C, E, B6, and B9 (folic acid)) also play a role in immunodeficiency [14]. 


\section{SOME FOODS THAT HELP BOOST THE IMMUNE SYSTEM}

\subsection{Nigella Sativa Oil}

Nigella sativa (black seed) seeds contain 32 to 40 percent fixed oil (Figure 1), which is rich in unsaturated and essential fatty acids. According to the chemical characteristics of the total lipids and the fatty acid profile, the essential unsaturated fatty acids are linoleic and oleic. Linoleic acid is the main component of omega- 6 fatty acids that reduces the risk of cardiovascular diseases. High intake of linoleic acid increases insulin sensitivity and reduces LDL cholesterol and the risk of hypertension. The fixed oil in Nigella sativa is also rich in $\beta$-sitosterol. According to the chemical analysis, 85 percent of the fixed oil is composed of unsaturated fatty acids. Nigella sativa seeds contain essential oils [thymoquinone (tq), thymohydroquinone (thq), dithymoquinone (nigellone), thymol, carvacrol, $\alpha$ and $\beta$-pinene, d-limonene, dihydrogeraniol, p-cymene], and bitter substances. Fourteen preclinical studies address the bronchodilation, antihistaminic, anti-inflammatory, and immunomodulatory effects of Nigella sativa in animal or cellular models of asthma models. Moreover, seven clinical trials point out improvements in asthma outcomes, such as symptoms, pulmonary function, and laboratory parameters (15).

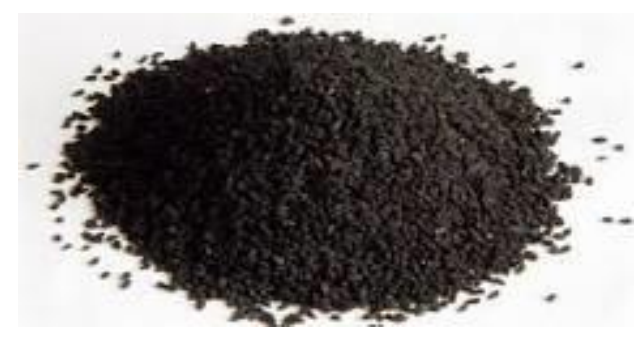

Figure 1. Nigella Sativa Seeds

Hussain and Hussain reported that Nigella sativa oil resulted in an improvement in 70 percent of allergic diseases, such as dust allergy, acne, neurodermatitis, asthma, and general immune system weakness among 600 patients in Germany (16).

The anticancer properties of Nigella sativa depend mainly on its potent anti-proliferative, proapoptotic, antioxidant, antimutagenic, and anti-metastatic effects. Its protective effects against tumor onset and progression also depend partly on its ability to suppress inflammation and boost the immune system (17).

Thymoquinone is the main active phenolic compound extracted from the essential oil of Nigella sativa seeds. It is widely used in the treatment of many diseases due to its high antioxidant properties. In vitro and in vivo studies suggest that it may have anti-inflammatory, antimicrobial, and anticancer properties. It is toxic only at high concentrations. It is an essential molecule due to its high biological activity and low systemic toxicity (18).

\subsection{Silverberry}

Ripe fruits and seeds of wild silverberry are rich in flavonoids, lycopene, carotenoids, phytosterols, and vitamins A, B1, B12, C, E, K, and P. Wild silverberry is, therefore, an 
excellent antioxidant that boosts the cardiovascular and immune system and protects against aging, inflammation, gastrointestinal ulcers, cancer, and radiation (Figure 2).

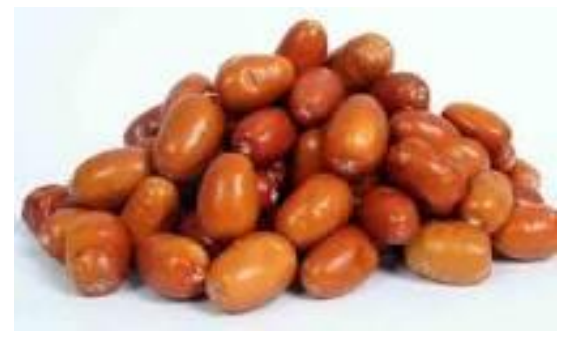

Figure 2. Silverberry

Silverberry is also a source of omega 7, that is, palmitoleic acid, which helps reduce the amount of cholesterol in the vessels and maintain the flexibility of the vessel walls. Palmitoleic acid is the source of the potential benefit of Omega 7. The human body can produce palmitoleic acid with a decreasing trend with age. Wild silverberry also has numerous health benefits for the cardiovascular and immune system because it is rich in antioxidants (flavonoids, lycopene, and carotenoids) and vitamins A, B1, B12, C, E, and K.

While omega 3 fights chronic inflammation, omega 7 serves a different purpose. It prevents arteriosclerosis in the endothelial layer, known as the vascular membrane, before the onset of disease. It enhances the communication between the muscle and fatty tissue and thus helps with the efficient use of energy. Even a small concentration of omega 7 puts a stop to the production of harmful fatty acids and adjusts the level of insulin. It reduces the risk of type-2 diabetes, prevents vascular stiffness, and controls the inflammatory markers known as C-reactive protein and associated with heart attacks and stroke. It increases HDL cholesterol levels and lowers LDL cholesterol levels. It is a powerful antioxidant and anti-inflammatory. It accelerates cellular regeneration and recovery and helps with the production of collagen, which plays a vital role in tissue repair.

Omega 7 prevents inflammation and boosts the immune system. It contains carotenoid antioxidant, which is greatly beneficial for the immune system. Wild silverberry is also thought to be effective against cancer cells because the antioxidants in it neutralize free radicals and protect against cancer [19].

\subsection{Date}

The date is an essential source of antioxidants as it is rich in phytochemicals (Figure 3). Antioxidants are known to be effective against chronic diseases, especially cardiovascular diseases, and cancer [20]. The date seed makes up 6-15 percent of its weight. The date seed extract is an essential antioxidant because it contains phenolic, flavonoid, catechin, and epicatechin (21). Date seed has ten times as high polyphenol content and antioxidant capacity as foods with high antioxidant capacity, such as grape seed (22). The date seed oil has more phenol than olive oil. There are two types of antioxidants; water-soluble (phenolic compounds and ascorbic acid) and fat-soluble (carotenoids, vitamin E) (23). The date is a good source of antioxidants because it is rich in antioxidant components and phenolic compounds consisting of p-coumaric, ferulic, and synaptic acids, and flavonoids, and procyanidins $(24,25)$. 


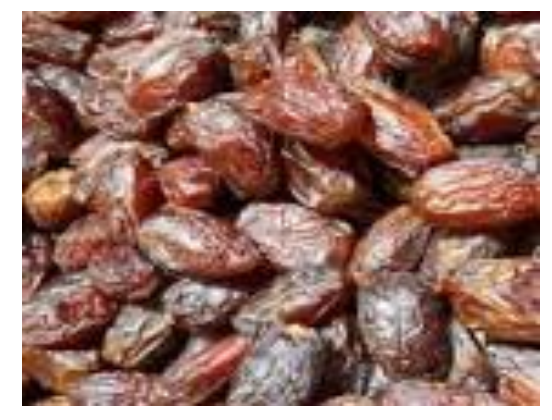

Figure 3. Date

Antioxidants in flavonoids reduce free radical activity and thus have positive effects against some chronic and cardiovascular diseases and some types of cancer.

The date is rich in phytochemicals. Vembu et al. (26) reported that date contains alkaloids, coumarin, tannins, flavonoids, phenolic compounds, and quinones. Of fat-soluble phytochemicals, date contains phytosterols the most. There are about 200 phytosterols, mostly in fruits and vegetables (27). The date has more phytoestrogens than various fruits (apple, orange, blueberry, and cranberry).

\subsection{Grape}

Grape is the second most extensively cultivated fruit crop in the world after orange. Substances in grape protect the body from infections, cancer, obesity, diabetes, and aging, and boost the immune and nervous system, and help relieve arthritis symptoms [28] (Figure 4). Grape contains vitamins, protein, carbohydrates, and minerals, and phenols and polyphenols (anthocyanin, flavanol, flavonol, phenolic acid, caffeic acid, catechin, quercetin, and resveratrol) and flavonoids, proanthocyanidins, and anthocyanidins [29,30]. Resveratrol is a stilbenoid and a phytoalexin, which is an antimicrobial compound synthesized in the leaves and grains of the grape. Resveratrol not only protects plants against diseases, but also has antifungal, antimicrobial, antitumor, and antioxidant effects in humans [31,32]. Its inflammatory markers increase the activity of antioxidant enzymes and suppress that of angiogenic and metastatic gene products [33]. Resveratrol is a powerful antioxidant that helps prevent cell damage caused by free radicals. [34,35]. Research shows that resveratrol inhibits the activity of some inflammatory enzymes [36].

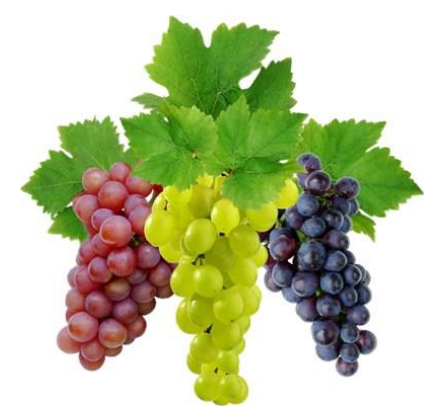

Figure 4. Grape 


\subsection{Asphodelus aestivus}

There is no data on the use of Asphodelus aestivus in modern medicine [37]. Asphodelus aestivus is vital for the defense system as it increases the WBC (white blood cells) count [38] (Figure 5) and has antimicrobial [39,40] and antioxidant effects [41]. Asphodelus aestivus is eaten cooked. However, there is no research on water-soluble vitamins and glutathione in Asphodelus aestivus.

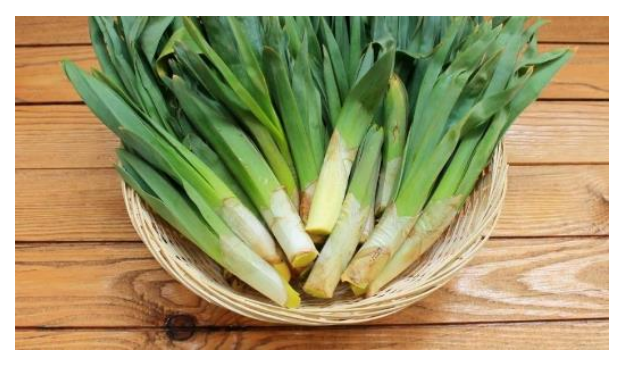

Figure 5. Asphodelus aestivus

\subsection{Rheum ribes}

Many antioxidative compounds in plants are free radical inhibitors, active oxygen scavengers, or in vitro reducing agents $[42,43]$. Rheum ribes (locally called $\iota s ̧$ gin) grows in the Eastern Anatolian Mountains, especially in Ağrı, Bingöl, Elazı $\breve{g}$, Hakkari, Kars, and Van regions. Its leaf stems are its edible parts consumed by locals, either raw or cooked [44,45] (Figure 6). Its leaves and stems of flowers taste bitter. It is effective against nausea, vomiting and constipation, and hemorrhoids, measles, and smallpox [46].

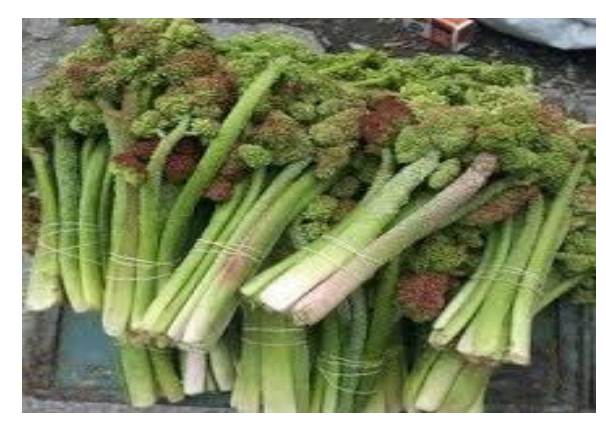

Figure 6. Rheum Ribes

\subsection{Carob}

Carob is rich in potassium $(970 \mathrm{mg} / 100 \mathrm{~g}$ dry substance) and calcium $(300 \mathrm{mg} / 100 \mathrm{~g}$ dry substance) as well as protein, amino acid, and sugar. Carob is not widely consumed, although it is rich in phytochemicals and other healthy substances, such as antioxidants, tannins, and polyphenols. However, it may become a functional food or a functional additive in the future [47] (Figure 7). Recent studies have shown that carob extracts have antioxidant activity and positive effects on human health, mainly to decrease the risk of cardiovascular diseases, diabetes, and cancer $[48,49]$. 


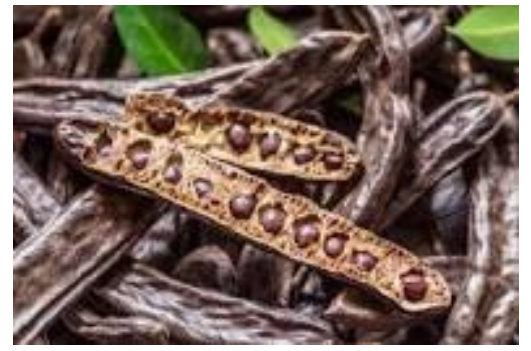

Figure 7. Carob

\section{CONCLUSIONS}

There is no cure yet for Covid-19. However, scientists and healthcare professionals recommend that people eat foods that are rich in vitamins and minerals to bolster their immune system to fight COVID-19 because immunodeficiency is the Achilles' heel of the Covid-19 pandemic.

People with chronic diseases, cardiovascular disease, kidney failure, diabetes, and cancer generally have a weakened immune system, and therefore, should consume foods that boost their immune system during outbreaks.

We, therefore, recommend that Nigella sativa oil, silverberry, date, grape, Asphodelus aestivus, Rheum ribes, and carob be consumed to boost the immune system.

\section{REFERENCES}

[1] Lu, R., Zhao, X., Li, J., Niu, P., Yang, B., Wu, H., Wang, W., Song, H., Huang, B., Zhu, N. (2020a). Genomic characterization and epidemiology of 2019novel coronavirus: implications for virus origins and receptor binding. Lancet, 395: 565-574.

[2] Lu, X., Chen, T., Wang, Y., Wang, J., Zhang, B., Li, Y., and Yan, F. (2020b). Adjuvant corticosteroid therapy for critically ill patients with COVID-19. medRxiv.https://doi.org/10.1101/2020.04.07.20056390.

[3] Del Rio, C. and Malani, P.N. (2020). COVID-19-new insights on a rapidly changing epidemic, JAMA, 323 : 1339-1340.

[4] Wu, Z. and McGoogan J.M. (2020). Characteristics of and Important Lessons From the Coronavirus Disease 2019 (COVID-19) Outbreak in China, JAMA, 323(13):1239-1242. doi:10.1001/jama.2020.2648

[5] Adalja A.A, Toner E, Inglesby T.V. (2020). Priorities for the US health community responding to COVID-19, JAMA, 323(14): 1343-1344.

[6] Caccialanza R, Laviano A, Lobascio F, Montagna E, Bruno R, Ludovisi S, Iacona I. (2020). Early nutritional supplementation in non-critically ill patients hospitalized for the 2019 novel coronavirus disease (COVID-19): Rationale and feasibility of a shared pragmatic protocol, Nutrition, 110: 835.

[7] Beck, M.A. (2007). Selenium and vitamin E status: impact on Viral Pathogenicity, The Journal of Nutrition, 137(5): 1338-1340. 
[8] Ritz, B.W. and Gardner, E.M. (2006). Malnutrition and energy restriction differentially affect viral immunity, The Journal of Nutrition, 136(5): 1141-1144.

[9] Kim, S.W. and Su, K.P. (2020). Using psychoneuroimmunity against COVID-19, Brain, Behavior, and Immunity Journal, 2: 1-2.

[10] http://pathmicro.med.sc.edu/ghaffar/innate.htm

[11] http://pathmicro.med.sc.edu/bowers/immune\%20cells.htm

[12] http://pathmicro.med.sc.edu/mayer/antigens2000.htm

[13] Aw, D., Silva, A. and Palmer, D. (2007). Immunosenescence: emerging challenges for an aging population, Immunology. 120 (4): 435-446.

[14] Chandra, R.K. (1997). Nutrition and the immune system: an introduction, American Journal of Clinical Nutrition, 66: 460-463.

[15] Koshak, A., Koshak, E. and Heinrich, M. (2017). Medicinal benefits of Nigella sativa in bronchial asthma: A literatüre review, Saudi Pharmaceutical Journal, 25(8): 1130-1136. doi: 10.1016/j.jsps.2017.07.002

[16] Hussain, D.A.S. and Hussain, M.M. (2016). Nigella sativa (black seed) is an effective herbal remedy for every disease except death - a Prophetic statement which modern scientists confirm unanimously: A review, Advancement in Medicinal Plant Research, 4(2): 27-57.

[17] Majdalawieh, A.F. and Fayyad, M.W. (2016). Recent advances on the anti-cancer properties of Nigella sativa, a widely used food additive, Ayurveda Integr Med., 7(3):173-180. doi: 10.1016/j.jaim.2016.07.004.

[18] Güzelsoy, P., Aydın, S. and Başaran, N. (2018). Potential Effects of Thymoquinone the Active Constituent of Black Seed (Nigella Sativa L.) on Human Health, Journal of Literature Pharmacy Sciences, 7-2 DOI:10.5336/PHARMSCI.2018-59816

[19] https://www.1faydalari.com/igdenin-faydalari/

[20] Alhaider IA, Mohamed ME, Ahmed KKM, Kumar AH. (2017). Date palm (Phoenix dactylifera) fruits as a potential cardioprotective agent: The role of circulating progenitor cells, Front Pharmacol, 8:592.

[21] Platat C, Habib HM, AL, Maqbali FD, Jaber NN, Ibrahim WH. (2014). Identification of date seeds varieties patterns to optimize the nutritional benefits of date seeds, Nutr Food Sci., 8: 1-8.

[22] Guendez R, Kallithraka S, Makris DP, Kefalas P. (2005). An analytical survey of the polyphenols of seeds of varieties of grape (Vitis Vinifera) cultivated in Greece: Implications for exploitation as a source of value-added phytochemical, Phytochem Analysis, 16: 17-23.

[23] Namiki M. (1990). Antioxidants/antimutagens in food, Crit Rev Food Sci Nutr,29: 273-300. 
[24] Al-Farsi M, Alasalvar C, Morris A, Baron M, Shahidi F. (2005). Comparison of antioxidant activity, anthocyanins, carotenoids and phenolics of three native fresh and sun-dried date (Phoenix dactylifera L.) varieties grown in Oman, J Agric Food Chem, 53: 7592-7599.

[25] Gu, L., Kelm, M.A., Hammerstone, J.F., Beecher, G., Holden, J. and Haytowitz, D. (2003). Screening of foods containing proanthocyanidins and their structural characterization, J Agric Food Chem., 3:51(25):7513-21. doi: 10.1021/jf034815d.

[26] Vembu S, Sivanasan D, Prasanna G. (2012). Effect Of Phoenix Deactylifera on High Fat Diet İnduced Obesity, J Chem Pharm Res.,4(1): 348-352.

[27] Bradford, P.G. and Awad, A.B. (2007). Phytosterols as anticancer compounds, Mol Nutr Food Res, 51(2): $161-170$.

[28] Percival, S.S., West, R.L. (2013). Effect of Health-Promoting Properties of Grapes, Including Resveratrol. In: Bioactives in Fruit: Health Benefits and Functional Fruits. Edit. By Skinner M. and Hunter, D., John Wiley Publ.

[29] Xia, E.Q., Deng G.F., Guo, Y.J., Li, H.B. (2010). Biological Activities of Polyphenols from Grapes. Int. J. of Molecular Sci., 622-646.

[30] Lim, T.K. (2013). Edible Medicinal and Non-Medicinal Plants. Volume 6, Fruits. Vitaceae: 450-482. Springer Science and Business Media Dordrecht.

[31] Clin Biochem (1997). Resveratrol:a molecule who setime has come and gone, Clin Biochem., 30(2):91-113. doi: 10.1016/s0009-9120(96)00155-5.

[32] Mizutani K, Ikeda K, Kawai Y, Yamori Y. (2001). Protective effect of resveratrol on oxidative damage in male and female stroke-prone spontaneously hypertensive rats. Clin Exp Pharma col Physiol, 28(1-2):55-59.

[33] Estrov Z, Shis ho di a S, Faderl S, Harris D, Van Q, Kantarji an H.M.(2003). Resveratrol blocks in terleu kin1 be ta-in du cedactivation of the nuclear transcription factor NF-kap paB, inhibits prolife ration,causes S-ph sear rest, and induces app to sis of acute my lo idle u like mi a cells. Blood, 102(3):987-95.

[34] Tedesco I, Russo M, Russo P, Iacomino G, Russo GL, Carraturo A. (2000). Antioxidant effect of red wine polyphe nols on red blood cells. J Nutr Biochem, 1(2):114-9.

[35] Mizutani K, Ikeda K, Kawai Y, Yamori Y. (2001). Protectve effect of resveratrol on oxidative damage in male and female stroke-pronespon taneously hypertensi ve rats. Clin Exp Pharmacol Physiol, 28(1-2):55-9.

[36] Lin JK, Tsa i SH. (1999). Chemopre vention of can cerand cardiovascular disease by resveratrol. Proc Natl Sci Counc Repub China B, 23(3):99-106.

[37] Polunin O., Huxley A., 1987. Flowers of the Mediterranean, Hogarth Press, London.

[38]http://www.google.com.tr/patents?id=MZAgAAAAEBAJ\&zoom=4\&dq=asphodelus\%20white20blood $\% 20$ $\%$ 20cell\&pg=PA1\#v=onepage \&q\&f=false $(21$ Ocak 2011) 
[39] Oskay M., Aktas K., Sari D., Azeri C. (2007). A comparative study of antimicrobial activity using well and disk diffusion method on Asphodelus aestivus (Liliaceae), Ekoloji, 16(62): 62-65.

[40] Tosun F., Akyüz Kızılay Ç., Sener B., Vural M., Palittapongarnpim P. (2004). Antimycobacterial Activity of Some Turkish Plants, Pharmaceutical Biology, 42: 39-43.

[41] Peksel A., Imamoglu S. (2009). Antioxidative properties of extracts from asphodelus aestivus brot (liliaceae), Annals of Nutrition and Metabolism, 55: 596-596.

[42] Duh, P.D. (1998). Antioxidant activity of burdock (Arctium lappa Linné): Its scavenging effect on freeradical and active oxygen, The Journal of the American Oil Chemists' Society, 75(4): 455-461.

[43] Gülçin, I., Mshvildadze, V., Gepdiremen, A. and Elias, R. (2006). The antioxidant activity of a triterpenoid glycoside isolated from the berries of Hedera colchica: 3-O-(beta-D-glucopyranosyl)-hederagenin, Phytother. Res., 20(2):130-4. doi: 10.1002/ptr.1821.

[44] Hedrick, U.P.(1972). Sturtevant's Edible Plants of the World, Dover Publications.

[45] Davis, P.H. (1965). Flora of Turkey, Edinburgh University Press. England.

[46] Baytop, T. (1984). Therapy with Medicinal Plants in Turkey (Past and Precent), Publications of the Istanbul University, No 3255, Turkey.

[47] Kumazawa S, Taniguchi M, Suzuki Y, Shimura M, Kwon MS, Nakayama T. (2002). Antioxidant activity of polyphenols in carob pods. J. Agric. Food Chem., 50: 373-377

[48] Loeb H, Vandenplas Y, Wursch P, Guesry P. (1989). Tannin-rich carob pod for the treatment of acute-onset diarrhea. J. Pediatr. Gastroenterol. Nutr., 8: 480- 485.

[49].Gruendel S, Garcia AL, Otto B, Mueller C, Steiniger J, Weickert MO, Speth M, Katz N, Koebnick C. (2006). Carob pulp preparation rich in insoluble dietary fiber and polyphenols enhances lipid oxidation and lowers postprandial acylated ghrelin in humans. J. Nutr., 136: 1533-1538. 